\title{
Cannabinoid WIN-55,212-2 mesylate inhibits ADAMTS-4 activity in human osteoarthritic articular chondrocytes by inhibiting expression of syndecan-1
}

\author{
YING KONG $^{1}$, WANCHUN WANG ${ }^{2}$, CHANGJIE ZHANG $^{1}$, YI WU $^{2}$, YANG LIU ${ }^{1}$ and XIAORONG ZHOU ${ }^{3}$ \\ Departments of ${ }^{1}$ Rehabilitation, ${ }^{2}$ Orthopedics and ${ }^{3}$ Geriatrics, \\ The Second Xiangya Hospital, Central South University, Changsha, Hunan 410011, P.R. China
}

Received May 15, 2015; Accepted February 23, 2016

DOI: $10.3892 / \mathrm{mmr} .2016 .5137$

\begin{abstract}
A central feature of osteoarthritis (OA) is the loss of articular cartilage, which is primarily attributed to cartilage breakdown. A group of metalloproteinases termed the A disintegrin and metalloproteinase with thrombospondin motifs (ADAMTS) family are reported to be important in cartilage breakdown. Recent studies have suggested that ADAMTS-4 is a major contributor to the pathogenesis of OA and that syndecan-1 is closely associated with activation of ADAMTS-4 in human chondrocytes. Accumulating evidence also suggests that cannabinoids have chondroprotective effects. The current study explored the effects of synthetic cannabinoid WIN-55,212-2 mesylate (WIN-55) on the expression of syndecan-1 and ADAMTS-4, as well as ADAMTS-4 activity, in unstimulated and interleukin (IL)- $1 \beta$-stimulated OA chondrocytes. Primary human OA articular chondrocytes were treated with WIN-55 in the presence or absence of IL-1 $\beta$ and cannabinoid receptor antagonists. The results of the present study demonstrated that WIN-55 inhibited ADAMTS-4 activity in unstimulated and IL-1 $\beta$-stimulated primary human OA articular chondrocytes in a concentration-dependent manner. Cannabinoid receptor type 1 (CB1) and 2 (CB2) were constitutively expressed in human $\mathrm{OA}$ articular chondrocytes. Furthermore, selective CB2 antagonist, JTE907, but not selective CB1 antagonist, MJ15, abolished the inhibitory effect of WIN-55 on ADAMTS-4 activity. WIN55 inhibited the expression of syndecan-1 but not ADAMTS-4, and overexpression of syndecan-1 reversed the inhibitory effect of WIN-55 on the ADAMTS-4 activity in unstimulated and IL- $1 \beta$-stimulated human OA articular chondrocytes. Despite having no
\end{abstract}

Correspondence to: Dr Xiaorong Zhou, Department of Geriatrics, The Second Xiangya Hospital, Central South University, 139 Remin Middle Road, Changsha, Hunan 410011, P.R. China

E-mail: xiaorong_xymu@sina.com

Key words: cannabinoid, WIN-55,212-2 mesylate, A disintegrin-like and metallopeptidase with thrombospondin motifs-4, syndecan-1, osteoarthritis, chondrocyte, cannabinoid receptor type 2 significant effect on syndecan-1 gene promoter activity, WIN-55 markedly decreased the stability of syndecan-1 mRNA via CB2. In conclusion, to the best of our knowledge, the present study provides the first in vitro evidence supporting that the synthetic cannabinoid WIN-55 inhibits ADAMTS-4 activity in unstimulated and IL-1 $\beta$-stimulated human OA articular chondrocytes by decreasing the mRNA stability/expression of syndecan-1 via CB2. This suggests a novel mechanism by which cannabinoids may prevent cartilage breakdown in OA. In addition, it also provides novel insights into the pharmacological effects of synthetic cannabinoids on OA.

\section{Introduction}

Osteoarthritis (OA) is the most common chronic degenerative joint disease, affecting millions of individuals worldwide and approximately 2.7 millions in the USA alone (1). Pro-inflammatory cytokines, including interleukin (IL)- $1 \beta$ and tumor necrosis factor (TNF)- $\alpha$, are critical in the development of OA by stimulating chondrocyte responses that promote catabolism of type II collagen and proteoglycans, thus, compromising cartilage extracellular matrix integrity and tissue homeostasis in OA (2). OA is characterized by progressive loss of articular cartilage and degradation of the cartilage matrix (3), which is primarily attributed to proteolysis of proteoglycans and collagens, the major structural components of cartilage matrix (4). Aggrecanases are a class of proteinases belonging to the A disintegrin and metalloproteinase with thrombospondin motifs (ADAMTS) family. Previous studies have demonstrated that ADAMTS-1, $-4,-5,-8,-9$, and -15 possess aggrecanase activity $(3,4)$. The degradation of aggrecan by aggrecanase-1 (also known as ADAMTS-4) is an early event in osteoarthritic cartilage damage (5). Song et al (6) reported that knockdown of ADAMTS-4 attenuates the degradation of aggrecan in human cartilage stimulated by TNF- $\alpha$ and oncostatin M. Other studies have demonstrated that ADAMTS-4 is selectively overexpressed in human OA cartilage and is positively correlated with the degree of cartilage destruction $(3,4)$, suggesting that ADAMTS-4 is an important aggrecanase in human OA cartilage and its activity is closely associated with the pathogenesis of OA. 
Syndecans are a family of cell-surface heparan sulfate proteoglycans comprising four members: Syndecan-1, -2, -3 and -4 (7). Syndecans interact with a variety of extracellular matrix molecules, growth factors and cytokines via their glycosaminoglycan chains $(5,8)$. Expression of all four syndecans has been observed in chondrocytes $(5,9)$. A previous study also demonstrated that ADAMTS-4 activation in human chondrosarcoma cells requires syndecan-1, suggesting that syndecan-1 is critical for the activation of ADAMTS-4 in human chondrocytes (10).

Cannabinoids, which have anti-inflammatory effects and reduce joint damage (11), predominantly function through $\mathrm{G}$ protein-coupled membrane receptors (12). Two major cannabinoid receptors, cannabinoid receptor type 1 (CB1) and 2 (CB2), are primarily expressed in the nervous and immune system, respectively (12). A recent report demonstrated that $\mathrm{CB} 1$ and $\mathrm{CB} 2$ are both expressed in human OA articular chondrocytes (11). Accumulating evidence suggests that cannabinoids have chondroprotective effects and may be useful for the treatment of OA $(11,13)$. Furthermore, it has been demonstrated that biologically stable synthetic cannabinoids have direct protective effects against cartilage matrix breakdown by reducing IL-1-induced proteoglycan and collagen degradation in bovine cartilage, potentially via $\mathrm{CB}$ receptors $(11,12)$.

The present study explored the effects of synthetic cannabinoid WIN-55,212-2 mesylate (WIN-55) on the expression of syndecan-1 and ADAMTS-4, as well as ADAMTS-4 activity, in unstimulated and IL-1 $\beta$-stimulated primary human OA articular chondrocytes.

\section{Materials and methods}

Chondrocyte culture and treatments. Primary human OA articular chondrocytes (cat no. 402OAK-05a) and human chondrocyte growth medium (cat no. 411-500) were purchased from Cell Applications, Inc. (San Diego, CA, USA). The cells were cultured in the growth medium supplemented with $5 \%$ fetal bovine serum (Thermo Fisher Scientific, Inc., Waltham, MA, USA) and $100 \mathrm{U} / \mathrm{ml}$ penicillin-streptomycin (Sigma-Aldrich, Beijing, China) in an incubator with a humidified atmosphere of $95 \%$ air and $5 \% \mathrm{CO}_{2}$ at $37^{\circ} \mathrm{C}$. The cells were cultured in the presence or absence of $10 \mathrm{ng} / \mathrm{ml}$ IL-1 $\beta$ (R\&D Systems, Inc., MN, USA) for $24 \mathrm{~h}$. Subsequently, the cells were treated with or without selective CB1 antagonist $\mathrm{MJ} 15(1,10$ or $50 \mu \mathrm{M}$; cat no. 4063; Tocris Bioscience, Bristol, UK) or selective CB2 antagonist JTE907 $(0.1,0.3$, or $0.6 \mu \mathrm{M}$; cat no. 2479; Tocris Bioscience) for $30 \mathrm{~min}$ prior to the addition of WIN-55 $(0.5,1.0$, 2.0, 4.0 or $8.0 \mu \mathrm{M}$; cat no. 1038 ; Tocris Bioscience) for a further $24 \mathrm{~h}$.

ADAMTS-4 activity assay. ADAMTS-4 activity was determined using a fluorimetric SensoLyte 520 Aggrecanase-1 Assay kit (cat no. AS-72114; AnaSpec, Inc., Fremont, CA, USA), according to the manufacturer's protocol $(14,15)$. Briefly, a fluorogenic substrate (5-FAM/TAMRA) was incubated with whole cell lysates $(25 \mu \mathrm{g}$ in $50 \mu \mathrm{l})$. Measurements were performed at $37^{\circ} \mathrm{C}$ over $90 \mathrm{~min}$ using a multiplate reader (SpectraMax Plus 384; Molecular Devices, LLC, Beijing, China) at excitation/emission wavelengths of $490 / 520 \mathrm{~nm}$. At the time of the assay, viable cells were counted using trypan blue and a a Levy Counting Chambers hemocytometer (Thomas Scientific Inc, Swedesboro, NJ, USA), as previously described (16). The measured fluorescence in the assay was normalized against the number of viable cells (per $10^{4}$ viable cells) to exclude the effects of IL-1 $\beta$ and WIN-55 on chondrocyte proliferation and survival.

Western blot analysis. Whole cell lysates were extracted by incubating the cells with lysis buffer [50 mM Tris/HCl, (pH7.2), $150 \mathrm{mM} \mathrm{NaCl}, 1 \%$ (v/v) Triton X-100, 1 mM sodium orthovanadate, $50 \mathrm{mM}$ sodium pyrophosphate, $100 \mathrm{mM}$ sodium fluoride, $0.01 \%(\mathrm{v} / \mathrm{v})$ aprotinin, $4 \mu \mathrm{g} / \mathrm{ml}$ pepstatin A, $10 \mu \mathrm{g} / \mathrm{ml}$ leupeptin and $1 \mathrm{mM}$ phenylmethanesulfonyl fluoride; all purchased from Sigma-Aldrich] on ice for $30 \mathrm{~min}$ and removal of cell debris by centrifugation at $2,000 \mathrm{x}$ for $15 \mathrm{~min}$ at $4{ }^{\circ} \mathrm{C}$. The protein concentration was determination by the Coomassie blue (Bio-Rad Laboratories, Inc., Hercules, CA, USA) method. Briefly, $50 \mathrm{ml}$ $0.1 \mathrm{M} \mathrm{NaOH}$ were added to each well and the plate was incubated at $37^{\circ} \mathrm{C}$ for $2 \mathrm{~h}$. Following incubation, $200 \mathrm{ml}$ of a 1:6 dilution of Coomassie blue were added to each well and left at room temperature for $30 \mathrm{~min}$. The absorbance was determined with a SmartSpec Plus spectrophotometer (Bio-Rad Laboratories, Inc.) at $405 \mathrm{~nm}$. Equal concentrations of protein for each sample were separated by $10 \%$ SDS-polyacrylamide gel electrophoresis (at $100 \mathrm{~V}$ for $90 \mathrm{~min}$ ) and blotted onto a polyvinylidene difluoride microporous membrane (EMD Millipore, Billerica, MA, USA). The membranes were blocked with $5 \%$ skim milk powder in Tris-buffered saline-Tween 20 (Sigma-Aldrich) for $2 \mathrm{~h}$ and subsequently incubated for $1 \mathrm{~h}$ with a 1:500 dilution of goat anti-human ADAMTS-4 polyclonal antibody (cat no. sc-16533), rabbit anti-human syndecan-1 polyclonal antibody (cat no. sc-5632) or mouse anti-human $\beta$-actin monoclonal antibody (cat no. sc-81178). The membranes were washed and proteins detected using bovine anti-goat (cat no. sc-2350), anti-rabbit (cat no. sc-2370) or anti-mouse (cat no. sc-2371) horseradish peroxidase-conjugated secondary antibody $(1: 5,000)$ for $1 \mathrm{~h}$. All antibodies were purchased from Santa Cruz Biotechnology, Inc. (Dallas, TX, USA). Peroxidase was detected using an enhanced chemiluminescence kit (GE Healthcare Life Sciences, Shanghai, China). Three independent experiments were performed.

Plasmid construction and stable transfection. Human syndecan-1 cDNA clone (cat no. SC118272) was purchased from OriGene Technologies, Inc. (Beijing, China) and the full-length syndecan-1 cDNA sequence was subcloned into a pcDNA $3.1(+)$ expression vector (Thermo Fisher Scientific, Inc.) at the KpnI and $\mathrm{XhoI}$ restriction sites, using the empty pcDNA 3.1 (+) vector as the vector control. The syndecan-1 expression vector was transfected into cells using Lipofectamine 2000 transfection reagent (Thermo Fisher Scientific, Inc.), according to the manufacturer's protocol. Pools of stable transfectants were generated via selection with G418 (600 $\mu \mathrm{g} / \mathrm{ml}$; Sigma-Aldrich), according to the manufacturer's protocol (Thermo Fisher Scientific, Inc.).

Reverse transcription-quantitative polymerase chain reaction $(R T-q P C R)$. RNA was prepared from cells using TRIzol reagent (Thermo Fisher Scientific, Inc.) followed by purification with the TURBO DNA-free System (Ambion; Thermo Fisher Scientific, Inc.). cDNA was synthesized using SuperScript II reverse transcriptase (Thermo Fisher Scientific, Inc.). RT-qPCR 
was performed using an ABI-PRISM 7700 Sequence Detection System (Applied Biosystems; Thermo Fisher Scientific, Inc.) and the fluorescent dye SYBR Green Master Mix (Thermo Fisher Scientific, Inc.) as described by the manufacturer. The primers used were as follows: Forward, 5'-GCCGCAAATTGT GGCTACT-3' and reverse, 5'-GCTGCGTGTCCTTCCAAGT-3' for syndecan-1; forward, 5'-GACTCATGACCACAGTCC ATGC-3' and reverse, 5'-AGAGGCAGGGATGATGTTCTG-3' for GAPDH. The PCR amplification conditions were as follows: $20 \mathrm{sec}$ at $95^{\circ} \mathrm{C}$ followed by 40 cycles of $3 \mathrm{sec}$ at $95^{\circ} \mathrm{C}$ and $30 \mathrm{sec}$ at $60^{\circ} \mathrm{C}$. Relative quantification of the level of the syndecan- 1 mRNA was determined using the $2^{-\Delta \Delta C q}$ method (17) and normalized against that of GAPDH in the same sample. Each experiment was repeated three times in duplicate.

Luciferase reporter assay. Cells were transfected with a commercially available human syndecan-1 promoter/luciferase reporter (cat no. S710408; SwitchGear Genomics, Shanghai, China) using Lipofectamine 2000 transfection reagent (Thermo Fisher Scientific, Inc.) and then treated with or without WIN-55 (8 $\mu \mathrm{M}$; Tocris Bioscience) for $24 \mathrm{~h}$. Luciferase assays were performed using the LightSwitch Luciferase Assay kit (cat no. LS010; SwitchGear Genomics) and the GloMax 96 Micorplate Luminometer (Promega Corporation, Madison, WI, USA), according to the manufacturer's protocol. Plasmid PRL-CMV (cat no. E2261; Promega Corporation) encoding Renilla reniformis luciferase was co-transfected with the reporter plasmid (at 1:5 molar ratio) in each transfection as an internal control for data normalization. Each experiment was repeated for three times in duplicate.

Measurement of mRNA stability. Cells were cultured in the presence or absence of $10 \mathrm{ng} / \mathrm{ml}$ IL-1 $\beta$ (R\&D Systems, Inc.) for $24 \mathrm{~h}$. Subsequently, the cells were treated with or without JTE907 $(0.6 \mu \mathrm{M}$; Tocris Bioscience) for $30 \mathrm{~min}$ prior to the addition of WIN-55 (1.0 or $8.0 \mu \mathrm{M}$; Tocris Bioscience) for a further 24-h incubation. Transcription inhibitor actinomycin D (1 $\mathrm{mg} / \mathrm{ml}$; Sigma-Aldrich) was added. The mRNA level of syndecan-1 was determined with RT-qPCR after 1, 2 and $4 \mathrm{~h}$ of actinomycin D treatment, and expressed as fold changes to mRNA level in control cells immediately prior to actinomycin D treatment (designated as 1).

Statistical analysis. Statistical analyses were performed using SPSS software for Windows (version 19.0; IBM SPSS, Armonk, NY, USA). All continuous variable values are expressed as the mean \pm standard deviation. Comparisons of means among multiple groups were performed with one-way analysis of variance followed by post-hoc pair-wise comparisons using Tukey tests. $\mathrm{P}<0.05$ was considered to indicate a statistically significant difference in this study.

\section{Results}

WIN-55 inhibits ADAMTS-4 activity via CB2 in unstimulated and IL-1 $\beta$-stimulated human OA articular chondrocytes. As demonstrated in Fig. 1, IL-1 $\beta$ stimulation increased the ADAMTS-4 activity by $\sim 5$ fold in human OA articular chondrocytes. Synthetic cannabinoid WIN-55 concentration-dependently inhibited the ADAMTS-4 activity in the

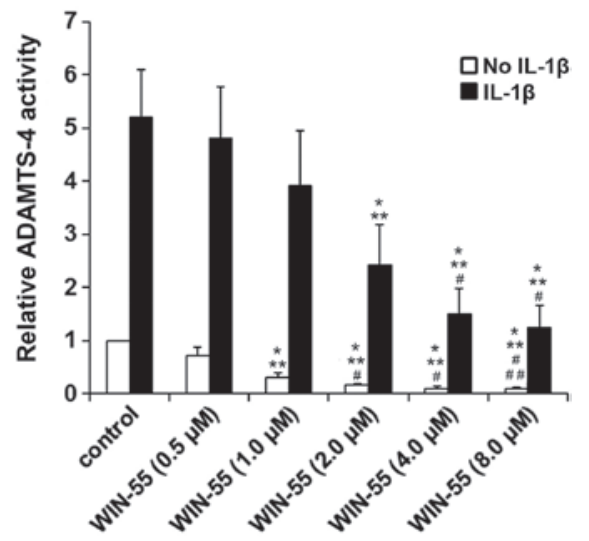

Figure 1. Effect of WIN-55 on ADAMTS-4 activity in human osteoarthritis (OA) articular chondrocytes in the presence or absence IL-1 $\beta$. Primary human OA articular chondrocytes were cultured in the presence or absence of $10 \mathrm{ng} / \mathrm{ml} \mathrm{IL}-1 \beta$ for $24 \mathrm{~h}$. The cells were subsequently treated with WIN-55 $(0.5,1.0,2.0,4.0$ or $8.0 \mu \mathrm{M})$ for $24 \mathrm{~h}$. ADAMTS-4 activity was measured with a fluorimetric SensoLyte 520 Aggrecanase-1 Assay and expressed as fold change to that of the unstimulated control (designated as 1). Data are presented as the mean \pm standard deviation. ${ }^{*} \mathrm{P}<0.05$ vs. control; ${ }^{* *} \mathrm{P}<0.05$ vs. WIN-55 $(0.5 \mu \mathrm{M}) ;{ }^{\#} \mathrm{P}<0.05$ vs. WIN-55 $(1.0 \mu \mathrm{M}) ;{ }^{\# \#} \mathrm{P}<0.05$ vs. WIN-55 $(2.0 \mu \mathrm{M})$. ADAMTS-4, A disintegrin and metalloproteinase with thrombospondin motifs-4; IL-1 $\beta$, interleukin-1 $\beta$; WIN-55, WIN-55,212-2 mesylate.

presence or absence of IL-1 $\beta(\mathrm{P}<0.05)$. The inhibitory effect of WIN-55 reached a plateau at $2 \mu \mathrm{M}$ in unstimulated cells ( $\sim 85 \%$ inhibition) and at $4 \mu \mathrm{M}$ in IL-1 $\beta$-stimulated cells $(\sim 70 \%$ inhibition). As presented in Fig. 2, classical cannabinoid receptors $\mathrm{CB} 1$ and $\mathrm{CB} 2$ were constitutively expressed in human OA articular chondrocytes. IL-1 $\beta$ stimulation demonstrated no significant effect on the expression of CB1 and CB2 ( $\mathrm{P}>0.05)$. As presented in Fig. 3, selective CB2 antagonist JTE907 concentration-dependently abolished the inhibitory effect of WIN-55 on the ADAMTS-4 activity in unstimulated and IL-1 $\beta$-stimulated human OA articular chondrocytes compared with WIN-55 treatment alone $(\mathrm{P}<0.05)$. Selective CB1 antagonist MJ15 did not cause any significant change to the effect of WIN-55 (P>0.05). Furthermore, MJ15 and JTE907 alone demonstrated no significant effect on ADAMTS-4 activity in the presence or absence of IL-1 $\beta$ compared with the control $(\mathrm{P}>0.05)$. The findings indicate that WIN-55 inhibits ADAMTS-4 activity via CB2 in unstimulated and IL-1 $\beta$-stimulated human OA articular chondrocytes.

WIN-55 inhibits the protein expression levels of syndecan-1, but not ADAMTS-4, in unstimulated and IL-1 $\beta$-stimulated human $O A$ articular chondrocytes. Western blot analyses were performed to examine the effect of WIN-55 on the expression of ADAMTS-4 in human OA articular chondrocytes. As presented in Fig. 4, IL-1 $\beta$ stimulation increased the expression of ADAMTS-4 by $\sim 3$ fold in human OA articular chondrocytes, compared with the unstimulated control $(\mathrm{P}<0.05)$. WIN-55 alone or in the presence of MJ15 or JTE907 demonstrated no significant effect on the expression of ADAMTS-4 in unstimulated or IL-1 $\beta$-stimulated cells $(\mathrm{P}>0.05)$. These findings indicate that WIN-55 inhibits ADAMTS-4 activity in human OA articular chondrocytes through mechanisms other than altering the protein expression of ADAMTS-4. 
A

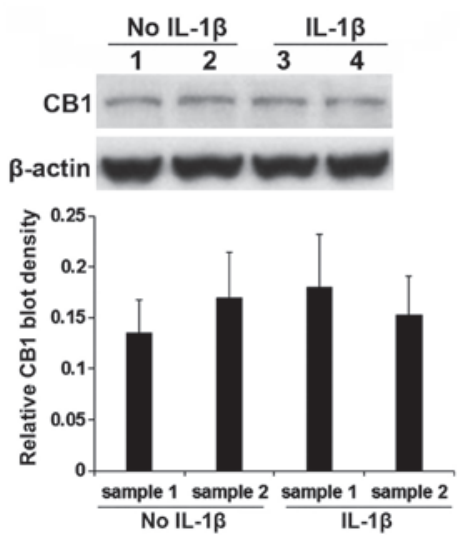

B

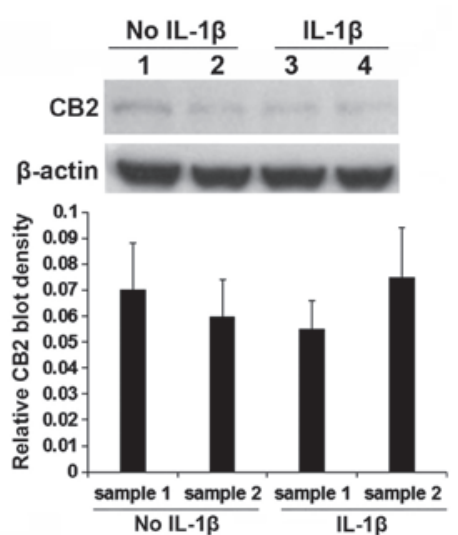

Figure 2. Expression of cannabinoid receptors in human osteoarthritis (OA) articular chondrocytes in the presence or absence of IL-1 3 . Primary human OA articular chondrocytes were cultured in the presence or absence of $10 \mathrm{ng} / \mathrm{ml} \mathrm{IL}-1 \beta$ for $24 \mathrm{~h}$. Expression of (A) CB1 and (B) CB2 were determined with western blot analyses using whole cell lysates from two independent preparations (samples 1 and 2): Lanes 1 and 3 , sample 1; lanes 2 and 4 , sample 2. $\beta$-actin was used as a loading control. The density of the CB1 and CB2 blots were normalized against that of the $\beta$-actin blot to obtain a relative blot density. Three independent experiments were performed for each western blot analysis. Data are presented as the mean \pm standard deviation. IL-1 $\beta$, interleukin-1 $\beta$; CB1/2, cannibinoid receptor type $1 / 2$.

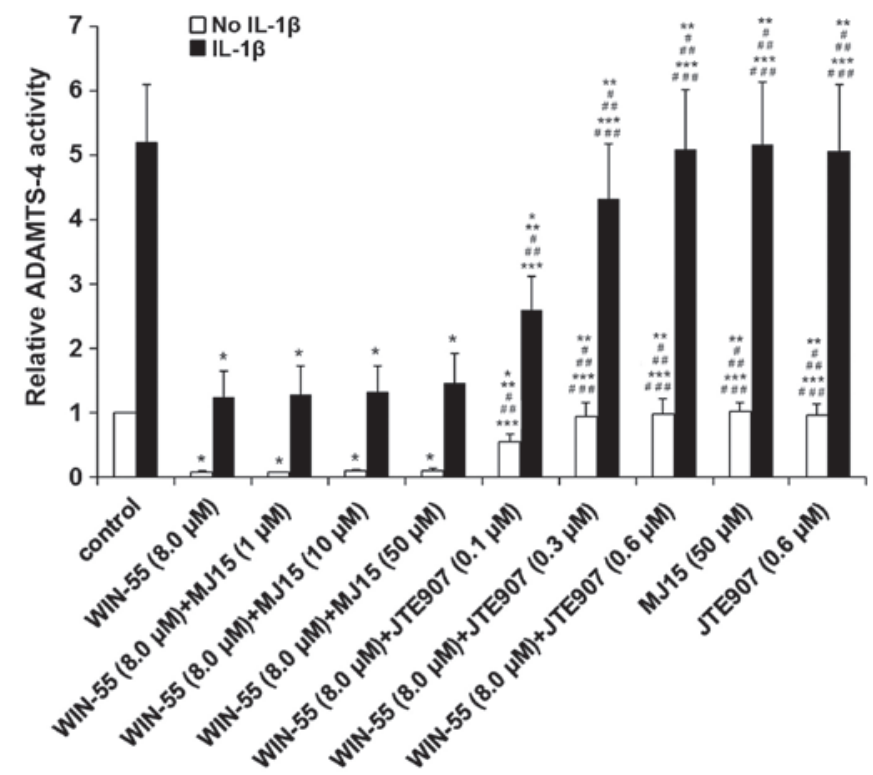

Figure 3. Effect of WIN-55 on ADAMTS-4 activity in human osteoarthritis (OA) articular chondrocytes in the presence or absence of IL-1 $\beta$ and cannabinoid receptor antagonists. Primary human OA articular chondrocytes were cultured in the presence or absence of $10 \mathrm{ng} / \mathrm{ml} \mathrm{IL-1 \beta}$ for $24 \mathrm{~h}$. The cells were treated with or without selective cannabinoid receptor type 1 (CB1) antagonist MJ15 $(1,10$ or $50 \mu \mathrm{M})$ or selective CB2 antagonist JTE907 (0.1, 0.3 or $0.6 \mu \mathrm{M})$ for 30 min prior to the addition of WIN-55 $(8.0 \mu \mathrm{M})$ for further 24-h incubation. ADAMTS-4 activity was measured with a fluorimetric SensoLyte 520 Aggrecanase-1 Assay and expressed as fold change to that of the unstimulated control (designated as 1). Data are presented as the mean \pm standard deviation. ${ }^{*} \mathrm{P}<0.05$ vs. control; ${ }^{* *} \mathrm{P}<0.05$ vs. WIN $-55 ;{ }^{~} \mathrm{P}<0.05$ vs. WIN-55 + MJ15 $(1 \mu \mathrm{M}) ;{ }^{\# \#} \mathrm{P}<0.05$ vs. WIN-55 + MJ15 $(10 \mu \mathrm{M}) ;{ }^{* * *} \mathrm{P}<0.05$ vs. WIN-55 + MJ15 $(50 \mu \mathrm{M}) ;{ }^{\# \#} \mathrm{P}<0.05$ vs. WIN-55 + JTE907 $(0.1 \mu \mathrm{M})$ ADAMTS-4, A disintegrin and metalloproteinase with thrombospondin motifs-4; IL-1 $\beta$, interleukin-1 $\beta$; WIN-55, WIN-55,212-2 mesylate.

As syndecan-1 is reportedly involved in the activation of ADAMTS-4 in human chondrosarcoma cells (10), the current study examined the effect of WIN-55 on the expression of syndecan-1 in human OA articular chondrocytes. As presented in Fig. 5A, IL-1 $\beta$ stimulation did not significantly alter the expression of syndecan-1 or the effect of WIN-55 on

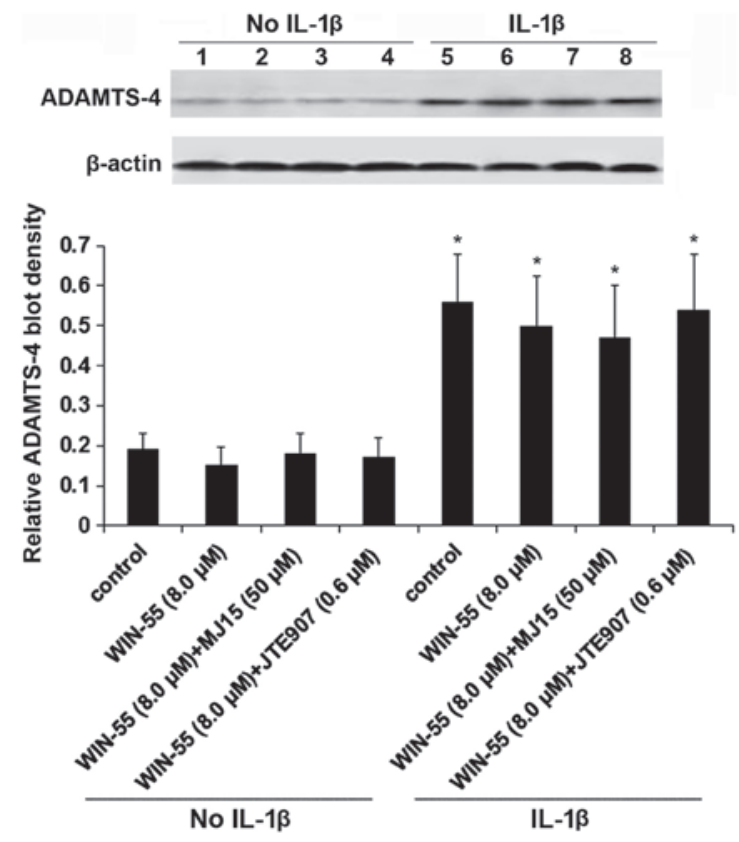

Figure 4. Expression of ADAMTS-4 in human osteoarthritis (OA) articular chondrocytes in the presence or absence of IL-1 $\beta$, WIN-55 and cannabinoid receptor antagonists. Primary human OA articular chondrocytes were cultured in the presence or absence of $10 \mathrm{ng} / \mathrm{ml} \mathrm{IL-1 \beta}$ for $24 \mathrm{~h}$. Then the cells were treated with or without selective cannabinoid receptor type 1 (CB1) antagonist MJ15 $(50 \mu \mathrm{M})$ or selective CB2 antagonist JTE907 $(0.6 \mu \mathrm{M})$ for $30 \mathrm{~min}$ prior to the addition of WIN-55 $(8.0 \mu \mathrm{M})$ for a further 24-h incubation. The expression of ADAMTS-4 was determined by western blot analyses: Lanes 1 and 5, control; lanes 2 and 6, WIN-55; lanes 3 and 7, WIN-55 + MJ15; lanes 4 and 8 , WIN-55 + JTE907. $\beta$-actin was used as a loading control. The density of the ADAMTS-4 blot was normalized against that of the $\beta$-actin blot to obtain a relative blot density. Three independent experiments were performed for each western blot analysis. Data are presented as the mean + standard deviation. ${ }^{*} \mathrm{P}<0.05$ vs. unstimulated control. IL-1 $\beta$, interleukin-1 $\beta$; ADAMTS-4, A disintegrin and metalloproteinase with thrombospondin motifs-4; WIN-55, WIN-55,212-2 mesylate.

syndecan-1 expression in human OA articular chondrocytes (P>0.05). WIN-55 decreased the constitutive protein level of syndecan-1 by $\sim 75 \%$ in unstimulated and IL-1 $\beta$-stimulated cells, as compared with the untreated controls. The effect of 
A
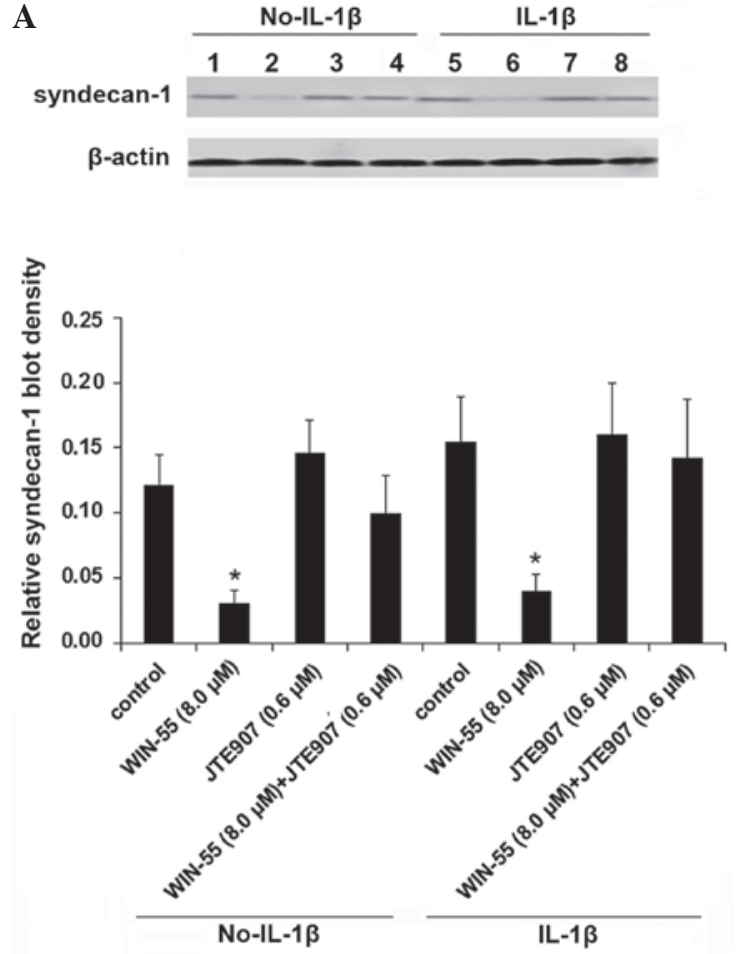

B

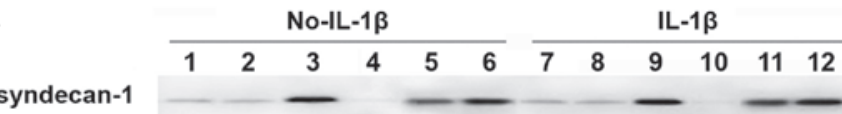

B-actin $\longrightarrow$

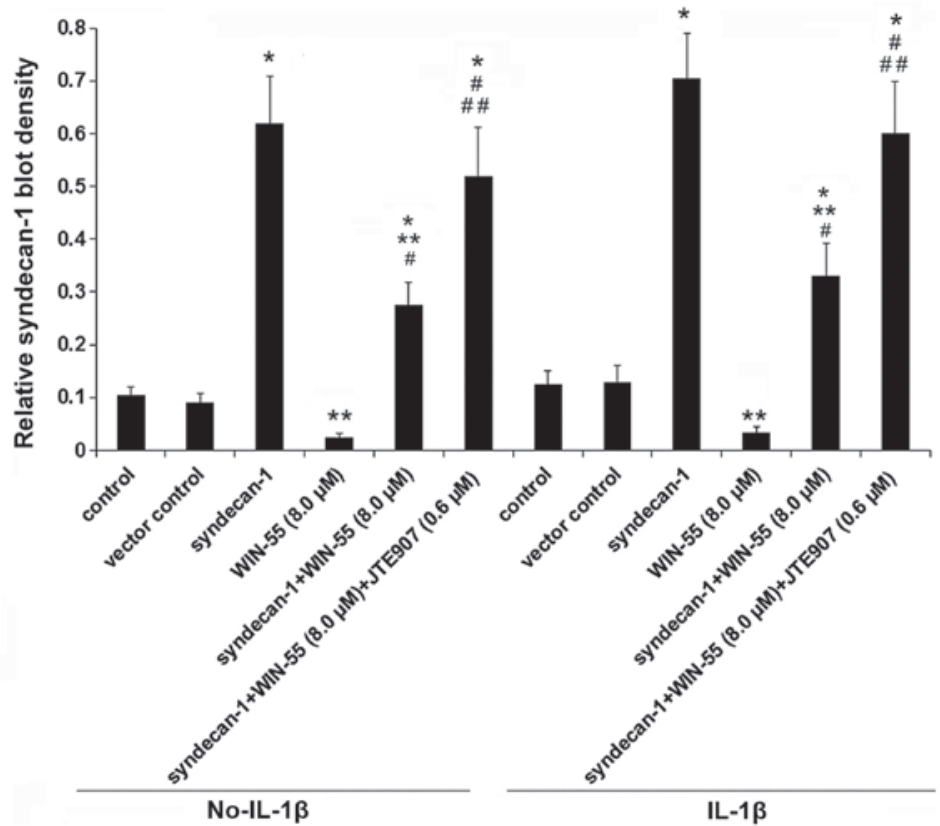

Figure 5. Protein expression levels of syndecan-1 in human osteoarthritis (OA) articular chondrocytes in the presence or absence of IL-1 $\beta$, WIN-55 and cannabinoid receptor type 2 (CB2) antagonist. Primary human OA articular chondrocytes were cultured in the presence or absence of 10 ng/ml IL-1 $\beta$ for 24 h. Then the cells were treated with or without selective CB2 antagonist JTE907 (0.6 $\mu \mathrm{M})$ for 30 min prior to the addition of WIN-55 (8.0 $\mu \mathrm{M})$ for a further 24 -h incubation. For syndecan-1 overexpression experiments, human OA articular chondrocytes were stably transfected with the pcDNA3.1-syndecan-1 plasmid. Cells stably transfected with the pcDNA3.1 plasmid were used as the vector control. The protein levels of syndecan-1 were determined by western blot analyses (A) Lanes 1 and 5, control; lanes 2 and 6, WIN-55 (8.0 $\mu \mathrm{M})$; lanes 3 and 7, JTE907 $(0.6 \mu \mathrm{M})$; lanes 4 and 8 , WIN-55 (8.0 $\mu \mathrm{M})+\mathrm{JTE} 907(0.6 \mu \mathrm{M})$. (B) Lanes 1 and 7, control; lanes 2 and 8 , vector control; lanes 3 and 9, syndecan-1 (overexpression); lanes 4 and 10, WIN-55 (8.0 $\mu \mathrm{M})$; lanes 5 and 11 , syndecan-1 + WIN-55 $(8.0 \mu \mathrm{M})$; lanes 6 and 12, syndecan-1 + WIN-55 $(8.0 \mu \mathrm{M})+$ JTE907 $(0.6 \mu \mathrm{M})$. $\beta$-actin was used as a loading control. Density of the syndecan-1 blot was normalized to that of the $\beta$-actin blot to obtain a relative blot density. Three independent experiments were performed for each western blot analysis. Data are presented as the mean \pm standard deviation. For panel (A): ${ }^{*} \mathrm{P}<0.05$ vs. control. For panel (B): ${ }^{*} \mathrm{P}<0.05$ vs. control and vector control; ${ }^{* *} \mathrm{P}<0.05$ vs. syndecan -1 ; ${ }^{\#} \mathrm{P}<0.05$ vs. WIN-55; ${ }^{\#} \mathrm{P}<0.05$ vs. syndecan-1 + WIN-55. IL-1 $\beta$, interleukin-1 $\beta$; WIN-55, WIN-55,212-2 mesylate.

WIN-55 treatment was completely abolished by CB2 antagonist JTE907 (Fig. 5A). Stable transfection of a human syndecan-1 expression vector resulted in a significant overexpression of syndecan-1 protein, which was inhibited by WIN-55 by $\sim 55 \%$, compared with control and vector control $(\mathrm{P}<0.05)$. JTE907 completely abolished the inhibitory effect of WIN-55 (Fig. 5B). A similar effect on syndecan-1 expression was observed at the mRNA level (Fig. 6). These findings indicate that WIN-55 exhibits no significant effect on the expression of ADAMTS-4, but does appear to inhibit the expression of syndecan-1 via CB2 in human OA articular chondrocytes.

Overexpression of syndecan-1 reverses the inhibitory effect of WIN-55/CB2 signaling on ADAMTS-4 activity in unstimulated and IL-1 $\beta$-stimulated human OA articular chondrocytes. As presented in Fig. 7, overexpression of syndecan-1 significantly increased ADAMTS-4 activity by 4.5 -fold in unstimulated cells and by 2.6 -fold in IL-1 $\beta$-stimulated human OA articular chondrocytes, compared with the control and vector control $(\mathrm{P}<0.05)$. Furthermore, overexpression of syndecan-1 completely reversed the inhibitory effect of WIN-55 on ADAMTS-4 activity, compared with WIN-55 treatment alone. In the presence of WIN-55, JTE907 significantly increased the inducing effect of syndecan-1 on ADAMTS-4 activity in unstimulated and IL-1 $\beta$-stimulated cells, as compared with syndecan-1 overexpression and WIN-55 treatment $(\mathrm{P}<0.05$; Fig. 7), although JTE907 alone showed no significant effect on ADAMTS-4 activity (Fig. 1). Taken together, the findings suggest that inhibiting the expression of syndecan-1 via CB2 may be a rate-limiting step for WIN55-induced inhibition of ADAMTS-4 activity in unstimulated and IL-1 $\beta$-stimulated human OA articular chondrocytes.

WIN-55 decreases the stability of syndecan-1 mRNA in unstimulated and IL-1 $\beta$-stimulated human OA articular chondrocytes. To examine whether WIN-55 decreases syndecan-1 mRNA levels in OA articular chondrocytes by transcriptionally inhibiting the syndecan-1 gene promoter, a human syndecan-1 gene promoter/luciferase reporter was transfected into human OA articular chondrocytes. As presented in Fig. 8, luciferase reporter assays demonstrated that IL-1 $\beta$ and WIN-55 did not exhibit any significant effect on syndecan-1 promoter activity, suggesting that WIN-55 treatment does not decrease syndecan-1 mRNA levels in human OA articular chondrocytes at the gene promoter/transcriptional level. The current study additionally examined the effect of WIN-55 on syndecan-1 mRNA stability. Following IL-1 $\beta$ stimulation, human OA articular chondrocytes were 


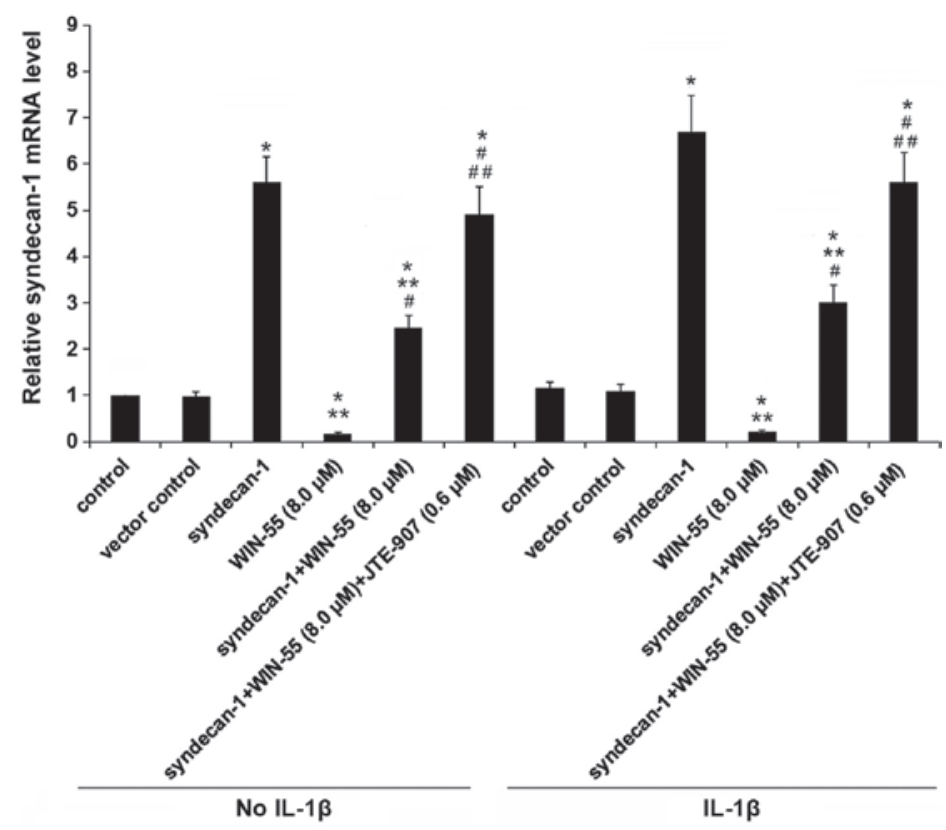

Figure 6. mRNA expression levels of syndecan-1 in human osteoarthritis (OA) articular chondrocytes in the presence or absence of IL-1 $\beta$, WIN-55 and cannabinoid receptor type 2 (CB2) antagonist. Primary human OA articular chondrocytes were cultured in the presence or absence of $10 \mathrm{ng} / \mathrm{ml} \mathrm{IL}-1 \beta \mathrm{for} 24 \mathrm{~h}$. Then the cells were treated with or without selective CB2 antagonist JTE907 $(0.6 \mu \mathrm{M})$ for $30 \mathrm{~min}$ prior to the addition of WIN-55 $(8.0 \mu \mathrm{M})$ for a further 24 -h incubation. For syndecan-1 overexpression experiments, human OA articular chondrocytes were stably transfected with the pcDNA3.1-syndecan-1 plasmid. Vector control cells were stably transfected with the pcDNA3.1 plasmid. The mRNA levels of syndecan-1 were determined by reverse transcription-quantitative polymerase chain reaction and expressed as fold change to that of the unstimulated control (designated as 1). Data are presented as the mean \pm standard deviation. ${ }^{*} \mathrm{P}<0.05$ vs. control and vector control; ${ }^{* *} \mathrm{P}<0.05$ vs. syndecan-1; ${ }^{\mathrm{P}} \mathrm{P}<0.05$ vs. WIN-55; ${ }^{\# \#} \mathrm{P}<0.05$ vs. syndecan- $1+$ WIN-55. WIN-55, WIN-55,212-2 mesylate; IL-1 $\beta$, interleukin-1 $\beta$.

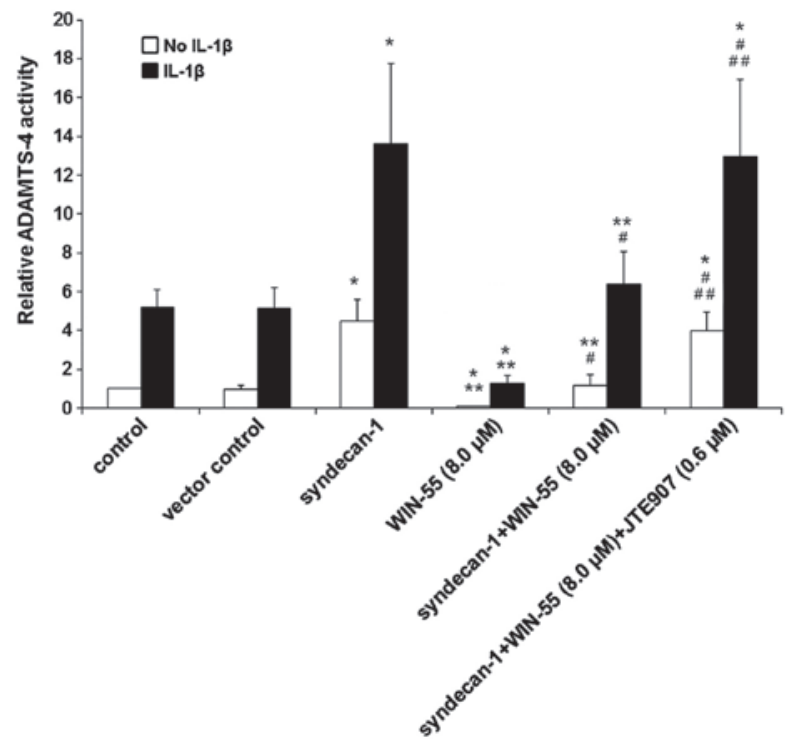

Figure 7. Effect of WIN-55 on ADAMTS-4 activity in human osteoarthritis (OA) articular chondrocytes in the presence or absence of IL-1 $\beta$, cannabinoid receptor type 2 (CB2) antagonist and overexpression of syndecan-1. Primary human OA articular chondrocytes were cultured in the presence or absence of $10 \mathrm{ng} / \mathrm{ml} \mathrm{IL-1 \beta}$ for $24 \mathrm{~h}$. Then the cells were treated with or without selective CB2 antagonist JTE907 $(0.6 \mu \mathrm{M})$ for $30 \mathrm{~min}$ prior to the addition of WIN-55 $(8.0 \mu \mathrm{M})$ for a further 24-h incubation. For syndecan-1 overexpression experiments, human OA articular chondrocytes were stably transfected with the pcDNA3.1-syndecan1 plasmid. Vector control cells were stably transfected with the pcDNA3.1 plasmid. ADAMTS-4 activity was measured with a fluorimetric SensoLyte 520 Aggrecanase-1 Assay and expressed as fold change to that of the unstimulated control (designated as 1). Data are presented as the mean \pm standard deviation. ${ }^{*} \mathrm{P}<0.05$ vs. control and vector control; ${ }^{* *} \mathrm{P}<0.05$ vs. syndecan $-1 ;{ }^{\#} \mathrm{P}<0.05$ vs. WIN-55; ${ }^{\# \#} \mathrm{P}<0.05$ vs. syndecan-1 + WIN-55. WIN-55, WIN-55,212-2 mesylate ADAMTS-4, A disintegrin and metalloproteinase with thrombospondin motif-4; IL-1 $\beta$, interleukin-1 $\beta$.

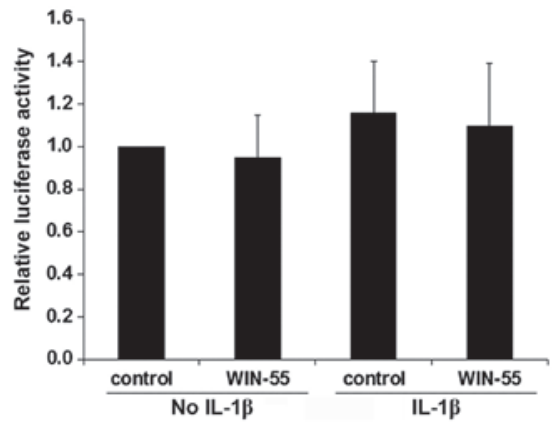

Figure 8. Effect of WIN-55 on human syndecan-1 gene promoter activity in human osteoarthritis (OA) articular chondrocytes in the presence or absence of IL-1 $\beta$. Primary human OA articular chondrocytes were transfected with human syndecan-1 gene promoter/luciferase reporter plasmids and then cultured in the presence or absence of $10 \mathrm{ng} / \mathrm{ml} \mathrm{IL}-1 \beta$ for $24 \mathrm{~h}$. Then the cells were treated with WIN-55 $(8.0 \mu \mathrm{M})$ for $24 \mathrm{~h}$. Luciferase activities were determined and expressed as fold change to that of the unstimulated control (designated as 1). Data are presented as the mean \pm standard deviation. WIN-55, WIN-55,212-2 mesylate; IL-1 $\beta$, interleukin-1 $\beta$.

treated with or without JTE907 for 30 min prior to the addition of WIN-55 for a further 24-h incubation period. The transcription inhibitor actinomycin $\mathrm{D}$ was then added and incubated with the cells for 1,2 or $4 \mathrm{~h}$. The mRNA level of syndecan-1 was subsequently determined using RT-qPCR and expressed as the fold change in mRNA levels of control cells at $0 \mathrm{~h}$ of actinomycin $\mathrm{D}$ treatment. Under actinomycin D treatment, WIN-55 dose-dependently decreased the syndecan-1 mRNA expression level compared with the control within $2 \mathrm{~h}$ of actinomycin $\mathrm{D}$ treatment $(\mathrm{P}<0.05$; Fig. 9). CB2 antagonism by JTE907 completely abolished 

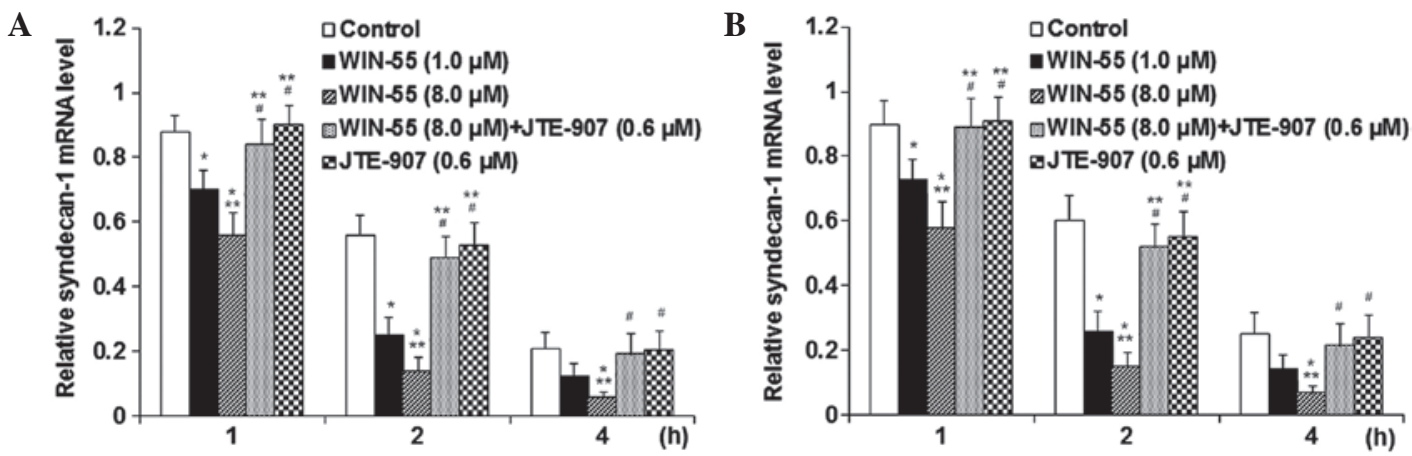

Figure 9. Effect of WIN-55 on syndecan-1 mRNA stability in human osteoarthritis (OA) articular chondrocytes in the presence or absence of interleukin (IL) $-1 \beta$ and cannabinoid receptor type 2 (CB2) antagonist. Primary human OA articular chondrocytes were cultured in the (A) absence or (B) presence of $10 \mathrm{ng} / \mathrm{ml} \mathrm{IL}-1 \beta$ for $24 \mathrm{~h}$. Then the cells were treated with or without selective CB2 antagonist JTE907 (0.6 $\mu \mathrm{M})$ for $30 \mathrm{~min}$ prior to the addition of WIN-55 $(1.0$ or $8.0 \mu \mathrm{M})$ for a further 24 -h incubation. Next, transcription inhibitor actinomycin D $(1 \mathrm{mg} / \mathrm{ml})$ was added and incubated with the cells for 1,2 or $4 \mathrm{~h}$. The mRNA level of syndecan-1 was determined by reverse transcription-quantitative polymerase chain reaction and expressed as fold change to that of the control cells immediately prior to actinomycin D treatment (designated as 1$)$. ${ }^{*} \mathrm{P}<0.05$ vs. control; ${ }^{* *} \mathrm{P}<0.05 \mathrm{vs}$. WIN-55 (1.0 $\left.\mu \mathrm{M}\right)$; ${ }^{*} \mathrm{P}<0.05 \mathrm{vs}$. WIN-55 (8.0 $\left.\mu \mathrm{M}\right)$. WIN-55, WIN-55,212-2 mesylate.

the effect of WIN-55, while JTE907 alone showed no significant effect on syndecan-1 mRNA levels compared with the control (Fig. 9). IL-1 $\beta$ stimulation did not significantly alter the effect of WIN-55 on the syndecan-1 mRNA level (Fig. 9). The findings suggest that WIN-55 decreases the expression of syndecan-1 in human OA articular chondrocytes by decreasing the stability of syndecan-1 mRNA via CB2; the effect of WIN-55 treatment was not significantly affected by IL-1 $\beta$ stimulation.

\section{Discussion}

Accumulating evidence suggests that cannabinoids have chondroprotective effects $(11,13)$. It has been demonstrated that biologically stable synthetic cannabinoids, including WIN-55, protect cartilage matrix from cytokine-induced degradation via $\mathrm{CB}$ receptors (12). A central feature of OA is the loss of articular cartilage. Cartilage breakdown is mediated by the complex interactions of pro-inflammatory cytokines, such as IL-1 $\beta$, inflammatory mediators, such as prostaglandin E2, and proteases, such as matrix metalloproteinases (13). It has become clear that proteolysis of the major constituents of the cartilage matrix, such as collagens and proteoglycans, is achieved not only by the classical matrix metalloproteinase family, but also by a relatively new group of metalloproteinases termed the aggrecanase/ADAMTS family. The degradation of aggrecan by aggrecanase-1 (also known as ADAMTS-4) is an early event in osteoarthritic cartilage damage (5). Recent studies have suggested that ADAMTS-4 is a major contributor to the pathogenesis of OA $(3,4)$. To the best of out knowledge, the present study provides the first evidence suggesting that cannabinoid WIN-55 inhibits the activity of ADAMTS-4 in OA chondrocytes.

The current study used IL- $1 \beta$-stimulated human OA articular chondrocytes as a cell model, as IL-1 $\beta$ is known to be important in the development of OA by promoting degradation of the cartilage matrix $(2,3)$. In the present study, 24-h treatment with 2 and $4 \mu \mathrm{M}$ WIN-55 inhibited ADAMTS-4 activity by $\sim 85$ and $70 \%$ in unstimulated and IL-1 $\beta$-stimulated human OA articular chondrocytes, respectively, suggesting that WIN-55 is a potent inhibitor of ADAMTS-4 activity in
OA chondrocytes. Considering that ADAMTS-4 is a major contributor to the pathogenesis of OA $(3,4)$, WIN-55 may have therapeutic value in the treatment of OA.

As an agonist of the classical cannabinoid receptors CB1 and CB2, WIN-55 has also been demonstrated to activate other receptors, including peroxisome proliferator activated receptor (PPAR)- $\alpha$ and $-\gamma(11,18-20)$. In agreement with a previous investigation (11), the current study observed that $\mathrm{CB} 1$ and $\mathrm{CB} 2$ were constitutively expressed in human OA articular chondrocytes. In the present study, a selective CB2 antagonist completely abolished the inhibitory effect of WN-55 on ADAMTS-4 activity, however, a selective CB1 antagonist exhibited no effect, indicating that WIN-55 inhibits ADAMTS-4 activity via CB2 in human OA articular chondrocytes. Although PPAR- $\alpha$ and $-\gamma$ are reportedly also expressed in human OA articular chondrocytes (11), these receptors were not investigated in the present study. In future studies, it may be important to investigate the role of PPAR receptors in WIN55-induced inhibition of ADAMTS-4 activity in OA articular chondrocytes.

In agreement with a previous investigation (4), the current study demonstrated that IL-1 $\beta$ induces the expression of ADAMTS-4 in human OA articular chondrocytes. Although WIN-55 demonstrated a potent inhibitory effect on ADAMTS-4 activity, it produced no direct effect on the expression of ADAMTS-4 in unstimulated and IL-1 $\beta$-stimulated human OA articular chondrocytes. Notably, WIN-55 inhibited the expression of syndecan-1, which is reportedly associated with the activation of ADAMTS-4 in human chondrosarcoma cells (10). Furthermore, overexpression of syndecan-1 reversed the inhibitory effects of WIN-55 on ADAMTS-4 activity, suggesting that inhibiting the expression of syndecan-1 is a rate-limiting step for WIN55-induced inhibition of ADAMTS-4 activity in human OA articular chondrocytes. Although no significant effect on the syndecan-1 gene promoter activity was observed, WIN-55 did significantly decrease the stability of syndecan-1 mRNA via CB2, which provides a mechanistic explanation for the inhibitory effect of WIN-55 on syndecan-1 expression in human OA articular chondrocytes. The mechanism by which WIN-55/CB2 signaling decreases syndecan-1 mRNA stability in OA articular chondrocytes should be explored in 
future studies. In addition, it was noted that IL-1 $\beta$ stimulation produced no significant effect on the WIN55-induced decrease in syndecan-1 mRNA stability. Similarly, IL-1 $\beta$ stimulation did not significantly alter the inhibitory effect of WIN-55 on syndecan-1 expression.

Syndecans are transmembrane heparan sulfate proteoglycans that interact with extracellular matrix molecules, growth factors and cytokines $(5,8)$. The syndecan family comprises four members: Syndecan-1, -2, -3 and -4 (7). As modulators of cellular activities, the syndecans are commonly associated with normal tissue remodeling or pathological changes in tissue organization (7). All four syndecans, and two major aggrecanases (ADAMTS-4 and -5), are expressed in chondrocytes $(5,10)$. Previous studies have suggested that the activation of ADAMTS-4 and -5 in chondrocytes requires syndecan-1 and -4 , respectively $(5,10)$. As the findings of the current study suggest that WIN-55 inhibits ADAMTS-4 activity in human OA articular chondrocytes by inhibiting the expression of syndecan-1, several questions arise for future studies: i) Can synthetic cannabinoids also alter the expression of syndecan-4 and regulate ADAMTS-5 activity in OA articular chondrocytes?; and i) as loss of articular cartilage is also a key pathological feature of rheumatoid arthritis (RA) $(11,21)$, can synthetic cannabinoids alter the expression of syndecans, in addition to the activities of ADAMTS, in RA articular chondrocytes and have potential therapeutic value for the treatment of RA?

In conclusion, the current study provides in vitro evidence supporting that synthetic cannabinoid WIN-55 inhibits ADAMTS- 4 activity in unstimulated and IL- $1 \beta$-stimulated human OA articular chondrocytes by decreasing the mRNA stability/expression of syndecan-1 via CB2. This suggests a novel mechanism by which cannabinoids may prevent cartilage breakdown in OA. In addition, it also provides novel insights regarding the pharmacological effects of synthetic cannabinoids on OA.

\section{References}

1. Lawrence RC, Felson DT, Helmick CG, Arnold LM, Choi H, Deyo RA, Gabriel S, Hirsch R, Hochberg MC, Hunder GG, et al; National Arthritis Data Workgroup: Estimates of the prevalence of arthritis and other rheumatic conditions in the United States: Part II. Arthritis Rheum 58: 26-35, 2008.

2. Goldring MB and Goldring SR: Osteoarthritis. J Cell Physiol 213: 626-634, 2007.

3. Xue J, Wang J, Liu Q and Luo A: Tumor necrosis factor- $\alpha$ induces ADAMTS-4 expression in human osteoarthritis chondrocytes. Mol Med Rep 8: 1755-1760, 2013.

4. Matsukawa T, Sakai T, Yonezawa T, Hiraiwa H, Hamada T, Nakashima M, Ono Y, Ishizuka S, Nakahara H, Lotz MK, et al: MicroRNA-125b regulates the expression of aggrecanase-1 (ADAMTS-4) in human osteoarthritic chondrocytes. Arthritis Res Ther 15: R28, 2013.
5. Echtermeyer F, Bertrand J, Dreier R, Meinecke I, Neugebauer K, Fuerst M, Lee YJ, Song YW, Herzog C, Theilmeier G and Pap T: Syndecan-4 regulates ADAMTS-5 activation and cartilage breakdown in osteoarthritis. Nat Med 15: 1072-1076, 2009.

6. Song RH, Tortorella MD, Malfait AM, Alston JT, Yang Z, Arner EC and Griggs DW: Aggrecan degradation in human articular cartilage explants is mediated by both ADAMTS- 4 and ADAMTS-5. Arthritis Rheum 56: 575-585, 2007.

7. Barre PE, Redini F, Boumediene K, Vielpeau C and Pujol JP: Semiquantitative reverse transcription-polymerase chain reaction analysis of syndecan- 1 and -4 messages in cartilage and cultured chondrocytes from osteoarthritic joints. Osteoarthritis Cartilage 8: 34-43, 2000.

8. Tkachenko E, Rhodes JM and Simons M: Syndecans: New kids on the signaling block. Circ Res 96: 488-500, 2005.

9. Molténi A, Modrowski D, Hott M and Marie PJ: Differential expression of fibroblast growth factor receptor-1, -2 , and -3 and syndecan-1, -2 , and -4 in neonatal rat mandibular condyle and calvaria during osteogenic differentiation in vitro. Bone 24: 337-347, 1999.

10. Gao G, Plaas A, Thompson VP, Jin S, Zuo F and Sandy JD: ADAMTS4 (aggrecanase-1) activation on the cell surface involves C-terminal cleavage by glycosylphosphatidyl inositol-anchored membrane type 4-matrix metalloproteinase and binding of the activated proteinase tochondroitin sulfate and heparan sulfate on syndecan-1. J Biol Chem 279: 10042-10051, 2004.

11. Dunn SL, Wilkinson JM, Crawford A, Le Maitre CL and Bunning RA: Cannabinoid WIN-55,212-2 mesylate inhibits interleukin- $1 \beta$ induced matrix metalloproteinase and tissue inhibitor of matrix metalloproteinase expression in human chondrocytes. Osteoarthritis Cartilage 2: 133-144, 2014.

12. Mbvundula EC, Bunning RA and Rainsford KD: Arthritis and cannabinoids: HU-210 and Win-55,212-2 prevent IL-1alpha-induced matrix degradation in bovine articular chondrocytes in-vitro. J Pharm Pharmacol 58: 351-358, 2006.

13. Dunn SL, Wilkinson JM, Crawford A, Le Maitre CL and Bunning RA. Cannabinoids: Novel therapies for arthritis? Future Med Chem 4: 713-725, 2012.

14. Moncada-Pazos A, Obaya AJ, Viloria CG, López-Otín C and Cal S: The nutraceutical flavonoid luteolin inhibits ADAMTS-4 and ADAMTS-5 aggrecanase activities. J Mol Med Berl 89: 611-619, 2011.

15. Tauchi R, Imagama S, Natori T, Ohgomori T, Muramoto A, Shinjo R, Matsuyama Y, Ishiguro N and Kadomatsu K: The endogenous proteoglycan-degrading enzyme ADAMTS-4 promotes functional recovery after spinal cord injury. J Neuroinflammation 9: 53, 2012.

16. Doyle A and Griffiths JB (eds): Cell and Tissue Culture: Laboratory Procedures. In: Cell Quantification. John Wiley \& Sons, Inc., Chichester, England, pp58-59, 1998.

17. Livak KJ and Schmittgen TD: Analysis of relative gene expression data using real-time quantitative PCR and the 2(-Delta Delta C(T)) Method. Methods 25: 402-408, 2001.

18. Pertwee RG, Howlett AC, Abood ME, Alexander SP, Di Marzo V, Elphick MR, Greasley PJ, Hansen HS, Kunos G, Mackie K, et al: International Union of Basic and Clinical Pharmacology. LXXIX. Cannabinoid receptors and their ligands: Beyond $\mathrm{CB}_{1}$ and $\mathrm{CB}_{2}$. Pharmacol Rev 62: 588-631, 2010.

19. Sun Y, Alexander SP, Kendall DA and Bennett AJ: Cannabinoids and PPARalpha signalling. Biochem Soc Trans 34: 1095-1097, 2006.

20. O'Sullivan SE and Kendall DA: Cannabinoid activation of peroxisome proliferator-activated receptors: Potential for modulation of inflammatory disease. Immunobiology 215: 611-616, 2010.

21. Goldring MB and Marcu KB: Cartilage homeostasis in health and rheumatic diseases. Arthritis Res Ther 11: 224, 2009. 